\title{
RESPONSE OF A MODEL OF CO OXIDATION WITH CO DESORPTION AND DIFFUSION TO A PERIODIC EXTERNAL CO PRESSURE
}

\author{
G. M.Buendía and E. Machado \\ Physics Department, Universidad Simón Bolívar, \\ Apartado 89000, Caracas 1080, Venezuela \\ P. A. Rikvold \\ Center for Materials Research and Technology, \\ School of Computational Science, and Department of Physics, \\ Florida State University, Tallahassee, Florida 32306-4120, USA
}

\begin{abstract}
We present a study of the dynamical behavior of a Ziff-Gulari-Barshad model with CO desorption and lateral diffusion. Depending on the values of the desorption and diffusion parameters, the system presents a discontinuous phase transition between low and high CO coverage phases. We calculate several points on the coexistence curve between these phases. Inclusion of the diffusion term produces a significant increase in the $\mathrm{CO}_{2}$ production rate. We further applied a square-wave periodic pressure variation of the partial CO pressure with parameters that can be tuned to modify the catalytic activity. Contrary to the diffusion-free case, this driven system does not present a further enhancement of the catalytic activity, beyond the increase induced by the diffusion under constant CO pressure.
\end{abstract}

\section{INTRODUCTION}

Phase transitions and critical phenomena in open nonequilibrium statistical systems have attracted a great deal of interest in recent decades, due to their wide applications in many branches of physics, chemistry, biology, economics, and even sociology [1]. Among these, surface reaction models have become the archetype to study out-of-equilibrium critical phenomena, and they have been intensely analyzed with the purpose of designing more efficient catalytic processes [2]. The Ziff, Gulari, Barshad (ZGB) model [3] with desorption (ZGB-k) [4, 5, 6, 7] describes some kinetic aspects of the reaction $\mathrm{CO}+\mathrm{O}_{2} \rightarrow \mathrm{CO}_{2}$ on a catalytic surface in terms of two parameters: the partial pressure of $\mathrm{CO}$, $y$, which represents the probability per unit of time that the next molecule arriving at the surface is a $\mathrm{CO}$, and the desorption parameter, $k$, which gives the probability per unit of time that a site occupied by a $\mathrm{CO}$ molecule is vacated. Below a critical CO desorption rate, the model exhibits a first-order phase transition between low and high coverage phases [4, 6, 6, [8]. A study of the metastable lifetimes associated with the transition between these phases indicates that the decay mechanism is very similar to the decay of metastable phases associated with equilibrium first-order phase transitions and can be described by the classic Kolmorogov-Johnson-Mehl-Avrami (KJMA) theory [9] of phase transformations by nucleation and growth 7]. Near the coexistence line between the active and the CO poisoned phase, the decay times of the metastable state are different if the ZGB-k model is driven into the active phase from the $\mathrm{CO}$ poisoned state or vice versa [7]. This asymmetry of the lifetimes, together with studies that indicate that it is possible to increase the efficiency of catalytic reactions by forcing the system by a periodic external pressure that switches back and forth around the discontinuous transition [10, 11, 12], inspired us to subject the system to a square-wave periodic CO pressure that stays for a time $t_{d}$ in the high-production region and $t_{p}$ in the low-production region. We showed that $t_{p}$ and $t_{d}$ can be tuned to significantly enhance the time-averaged catalytic activity of the system [6].

Many experiments indicate that at intermediate and high temperatures adsorbed CO is fairly mobile, while oxygen is still relatively immobile [5, 10]. In this work we aim at a more realistic process by incorporating CO diffusion into the ZGB-k model. The model that includes CO desorption and diffusion will be denoted the ZGB-(k,d) model. We investigate how the diffusion process affects the first-order kinetic phase transition and the response of the system to a periodic variation of the CO pressure.

The rest of this paper is organized as follows: in Sec. II we define the model and describe the Monte Carlo simulation techniques used. In Sec. III we present and discuss the results obtained when subjecting the model to a periodic external CO pressure. Finally, we present our conclusions in Sec. IV. 


\section{MODEL AND SIMULATIONS}

The ZGB-(k,d) model is simulated on a square lattice of linear size $L$ that represents the catalyst surface. A Monte Carlo simulation generates a sequence of trials: CO diffusion with probability per unit of time $d$, and adsorption or desorption with $(1-d)$. In nature, the diffusion process occurs more frequently than the adsorption/desorption process, so that $d \in(1 / 2,1)[13]$. The chemical processes include $\mathrm{CO}$ or $\mathrm{O}_{2}$ adsorption with probability per unit of time $1-k$, and $\mathrm{CO}$ desorption with probability per unit of time $k$. For the adsorption, a $\mathrm{CO}$ or $\mathrm{O}_{2}$ molecule is selected with probability per unit of time $y$ and $1-y$ respectively. The algorithm works in the following way. A site $i$ is selected at random. In the case of CO diffusion, if $i$ is empty or occupied by an $\mathrm{O}$ atom, the trial ends. Otherwise, a nearest neighbor of $i$ is selected at random; if it is vacant, the CO molecule exchanges with it, and reacts if any of its nearest-neighbors is an $\mathrm{O}$ atom, producing a $\mathrm{CO}_{2}$ molecule and leaving two empty sites on the surface. In the case of desorption, if $i$ is occupied by a CO, the site $i$ is vacated and the trial ends; if not, the trial also ends. In the case of adsorption, if a CO molecule is selected it can be adsorbed at the empty site $i$ if none of its nearest neighbors are occupied by an $\mathrm{O}$ atom. Otherwise, one of the occupied $\mathrm{O}$ neighbors is selected at random and removed from the surface, leaving $i$ and the selected neighbor vacant. $\mathrm{O}_{2}$ molecules require a nearest-neighbor pair of vacant sites to adsorb. Once the $\mathrm{O}_{2}$ molecule is adsorbed, it is dissociated into two $\mathrm{O}$ atoms. If an $\mathrm{O}$ atom is located next to a site filled with a $\mathrm{CO}$ molecule, they react to form a $\mathrm{CO}_{2}$ molecule that escapes, leaving two sites vacant. This process simulates the $\mathrm{CO}+\mathrm{O} \rightarrow \mathrm{CO}_{2}$ surface reaction. Since the diffusion is much faster than the other chemical processes, we will assume that the diffusion does not slow down the time scale for adsorption/desorption, which is the physically relevant time scale for the phase transformation [14]. Thus, we define the time unit as one Monte Carlo Step per Site, MCSS, in which the system performs, on average, one adsorption/desorption attempt per site.

\section{RESULTS}

Our simulations are realized on a square lattice of $100 \times 100$ sites, assuming periodic boundary conditions. We calculate the coverages $\theta_{\mathrm{CO}}$ and $\theta_{\mathrm{O}}$, defined as the fraction of surface sites occupied by CO and O, respectively, and $R_{\mathrm{CO}_{2}}$ defined as the rate of production of $\mathrm{CO}_{2}$. In Fig. 1 we show, for a particular value of $k$ and $d$, the dependence of the average values of the coverages and the production rate: $\left\langle\theta_{\mathrm{CO}}\right\rangle,\left\langle\theta_{\mathrm{O}}\right\rangle$, and $\left\langle R_{\mathrm{CO}_{2}}\right\rangle$, respectively, for an external constant CO pressure $y$. There are two inactive regions, $y<y_{1}\left(y_{1}\right.$ appears to be fairly independent of $k$ and $\left.d[7]\right)$, and $y>y_{2}(k, d)$. These correspond to the cases in which the surface is saturated with $\mathrm{O}$ and CO, respectively. In between, for $y_{1}<y<y_{2}(k, d)$, there is an active window where the system produces $\mathrm{CO}_{2}$. Notice that the maximum value of $\left\langle R_{\mathrm{CO}_{2}}\right\rangle$ is reached as $y_{2}(k, d)$ is approached from the low- $\theta_{\mathrm{CO}}$ phase. Fig. 1(b) shows that the effect of the $\mathrm{CO}$ diffusion is to widen the range of values of $y$ for which there is catalytic activity, producing a significant increase of the maximum $\mathrm{CO}_{2}$ production rate. In Fig. 2 we plot the coexistence point, $y_{2}$, as a function of $k$ for several values of $d$. The effect of the diffusion is to shift the transition to higher values of $y_{2}$, while maintaining approximately the same dependence of $y_{2}$ on $k$ that is observed at $d=0$.

Next, we perturb the system by applying an oscillating pressure $y(t)$ that in a period $T=t_{d}+t_{p}$ takes the values ,

$$
y=\left\{\begin{array}{ll}
y_{l} & \text { during the time interval } t_{d} \\
y_{h} & \text { during the time interval } t_{p}
\end{array},\right.
$$

located at both sides of the transition point, i.e., $y_{l}<y_{2}(k)<y_{h}$ (see Fig. [3] (a)).

We found that, for each choice of $y_{l}$ and $y_{h}$, we can vary the productivity of the system by tuning $t_{d}$ and $t_{p}$, the times that the driving force spends in the high and low coverage regions, respectively. In Fig. 31(b) it can be seen that the response to the periodic pressure shown in Fig. B(a), the $\mathrm{CO}_{2}$ production rate $R_{\mathrm{CO}_{2}}$, also exhibits an oscillatory behavior. We define the period-averaged value of $R_{\mathrm{CO}_{2}}$,

$$
r=\frac{1}{T} \int R_{\mathrm{CO}_{2}}(t) d t
$$

as the dynamic order parameter. For the parameters used in Fig. 3 (selected after an exhaustive search looking for the maximum productivity) the long-time average of $r,\langle r\rangle$ is almost the same as the maximum average $\mathrm{CO}_{2}$ production rate for constant $y$. In contrast, the ZGB-k model shows a marked enhancement of the catalytic activity when subjected to this type of periodic perturbation [6]. We think that this significant difference may possibly be due to the fact that the diffusion term modifies the decay mechanisms of the system near the transition. Previous studies in a lattice-gas model near an equilibrium first-order phase transition show that at high diffusion rates the nucleation no longer can be described by the classical KJMA theory [14]. The fact that applying a periodic CO pressure to the 
system with diffusion, does not seem to increase the $\mathrm{CO}_{2}$ production further, may indicate that the optimal mixing of $\mathrm{CO}$ and $\mathrm{O}$ on the surface was already reached by the inclusion of diffusion.

Depending on the values of $t_{d}$ and $t_{p}$, the system behavior defines two regions: a productive one with $r \neq 0$, and a non-productive one with $r \approx 0$, separated by a coexistence curve. In Fig. 团 we show a density plot of the average productivity $\langle r\rangle$ in terms of $t_{d}$ and $t_{p}$ for a particular choice of $y_{l}$ and $y_{h}$. Notice that the highest productivity is reached close to the coexistence curve. The plot suggests, as is also the case in the ZGB-k model [6], the existence of a dynamic phase transition (DPT), where the period-averaged order parameter $r$ takes the value $r \approx 0$ or $r \neq 0$ depending on whether the system is in a nonproductive state or in a productive one. A more detailed study of the nature of the transition, based on a finite-size scaling analysis of the fluctuations of the order parameter, is in progress and will be published elsewhere.

\section{CONCLUSIONS}

In this paper we have studied the ZGB model with CO desorption and diffusion, the ZGB-(k,d) model, and its dynamic response to periodic variations of the partial CO pressure. We found that, like the model without diffusion (ZGB-k), this system exhibits a non-equilibrium discontinuous phase transition between low and high CO coverage phases. The transition occurs at a $k$-dependent value of the $\mathrm{CO}$ pressure $y_{2}$. The effect of the diffusion term is to shift the transition to a higher pressure, such that $y_{2}(k, d)>y_{2}(k, 0)$, and to produce a significant increase of the maximum $\mathrm{CO}_{2}$ production rate. We further subjected the system to a square-wave periodic $\mathrm{CO}$ pressure that switches back and forth around the coexistence value that separates the low and high CO-coverage phases, staying for a time $t_{d}$ in the high-production region and for a time $t_{p}$ in the low production one. We found that $t_{p}$ and $t_{d}$ can be tuned to vary the catalytic activity of the system. However, contrary to the case of the ZGB-k model, the increase in the production of $\mathrm{CO}_{2}$ obtained in this way is negligible. We believe that this result indicates that the decay mechanisms of the metastable phase may be strongly affected by the diffusion process. We also found indications that this driven nonequilibrium system could have a dynamic phase transition between a dynamic phase of high $\mathrm{CO}_{2}$ production and a nonproductive phase. A more detailed study that includes the application of finite-size scaling techniques is necessary to establish the full details of the transition.

\section{Acknowledgments}

This work was supported in part by U.S. National Science Foundation Grant No. DMR-0240078 at Florida State University.

[1] H. J. Jensen, Self-Organized Criticality: Emergent Complex Behavior in Physical and Biological Systems, Cambridge University Press, Cambridge, England, (1998); J. Marro and R. Dickman, Non-equilibrium Phase transitions in Lattice Models, Cambridge University Press, Cambridge, England, (1999).

[2] K. Christmann, Introduction to Surface Physical Chemistry, Steinkopff Verlag, Darmstadt (1991); V.P.Z. Zhdanov and B.Kazemo, Surf. Sci. Rep. 20, 111 (1994); G. C. Bond Catalysis: Principles and Applications, Clarendon, Oxford (1987).

[3] R. M. Ziff, E. Gulari, and Y. Barshad, Phys. Rev. Lett. 56, 2553 (1986).

[4] T. Tomé and R. Dickman, Phys. Rev. E 47, 948 (1993).

[5] M. Ehsasi, M. Matloch, O. Frank, J. H. Block, K. Christmann, F. S. Rys, and W. Hirschwald, J. Chem. Phys. 91, 4949 (1989).

[6] E. Machado, G. M. Buendía, and P. A. Rikvold, Phys. Rev. E 71, 031603 (2005).

[7] E. Machado, G. M. Buendía, P. A. Rikvold, and R. M. Ziff, Phys. Rev. E 71, 016120 (2005).

[8] R. M. Ziff and B. J. Brosilow, Phys. Rev. A 46, 4630 (1992).

[9] A. N. Kolmogorov, Bull. Acad. Sci. USSR, Phys. Ser. 1, 335 (1937); W. A. Johnson and R. F. Mehl, Trans. Am. Inst. Mining Metall. Eng. 135, 416 (1939); M. Avrami, J. Chem. Phys. 7, 1103 (1939); 8, 212 (1940); 9, 177 (1941).

[10] R. Imbihl and G. Ertl, Chem. Rev. 95, 697 (1995).

[11] A. C. Lopez and E. V. Albano, J. Chem. Phys. 112, 3890 (2000).

[12] D. Hua and Y. Ma, Phys. Rev. E 66, 066103 (2002).

[13] N. Pavlenko, J. W. Evans, D. Liu, and R. Imbihl, Phys. Rev. E 65, 016121 (2001).

[14] S. Frank, D. E. Roberts, and P. A. Rikvold, J. Chem. Phys. 122, 064705 (2005). 


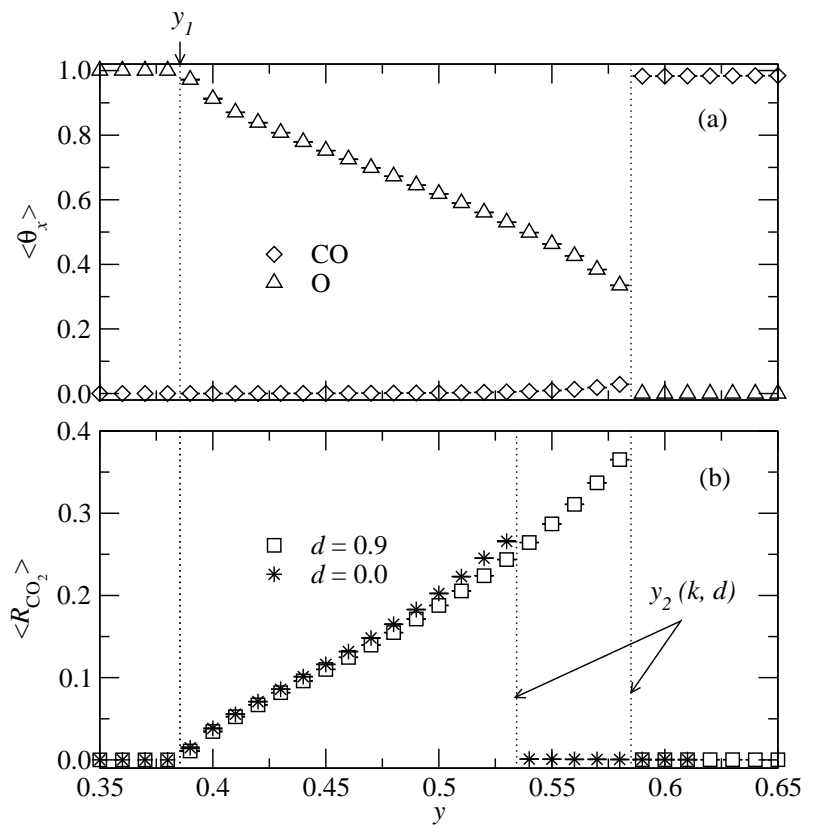

FIG. 1: (a) Average values of the $\mathrm{CO}$ and $\mathrm{O}$ coverages, $\theta_{\mathrm{CO}}$ and $\theta_{\mathrm{O}}$, shown as functions of the stationary applied CO pressure $y$, for $L=100$ with $k=0.01$ and $d=0.9$. A continuous, nonequilibrium phase transition occurs at $y_{1}$, and a discontinuous one at $y_{2}(k, d)$. (b) Comparison between the average $\mathrm{CO}_{2}$ production rate $\left\langle R_{\mathrm{CO}_{2}}\right\rangle$ at $d=0.9$ and $d=0$, calculated for the same values of $L$ and $k$ as in (a). Both $y_{2}$ and the maximum value of $\left\langle R_{\mathrm{CO}_{2}}\right\rangle$ increase significantly with $d$.

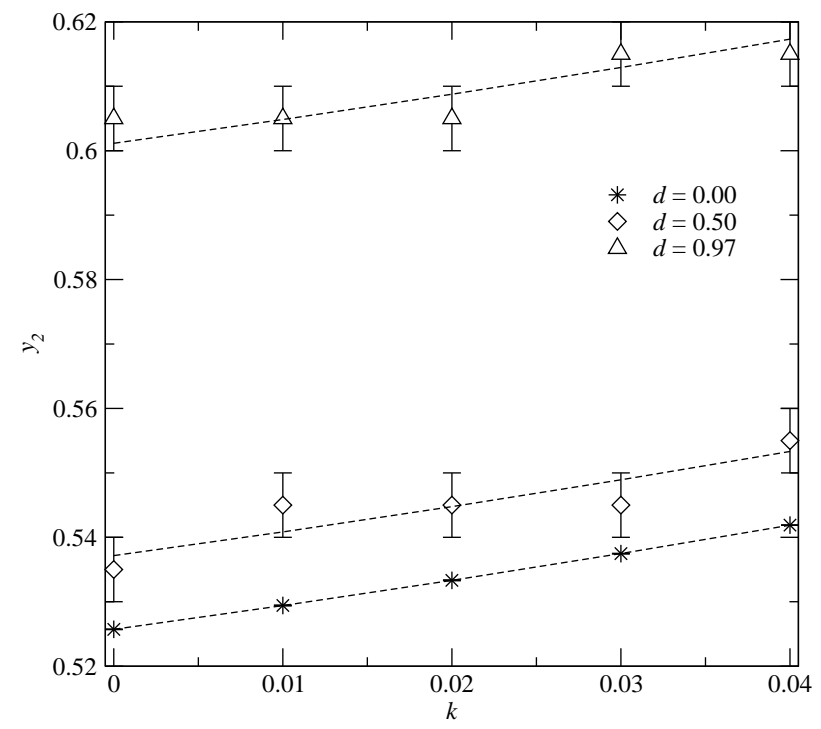

FIG. 2: Some points on the coexistence curve for several values of the diffusion probability per unit of time $d$. This is analogous to the pressure vs temperature phase diagram for a fluid in equilibrium. The continuous dashed lines represent a quadratic fit to the data. 

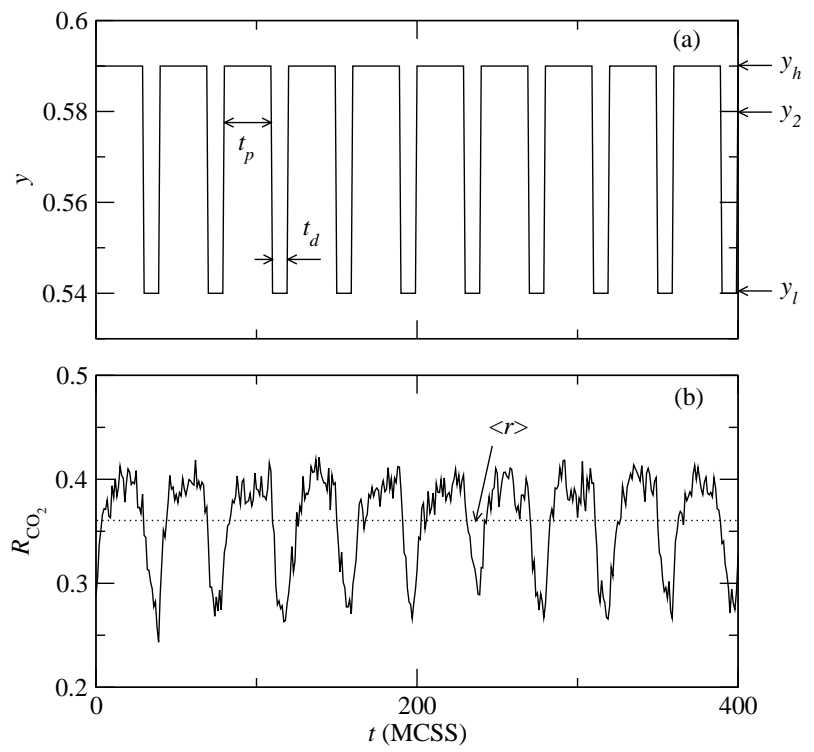

FIG. 3: (a) Applied periodic pressure of CO, $y(t)$, which takes the values $y_{l}=0.54$ and $y_{h}=0.59$ during the time intervals $t_{d}=10$ and $t_{p}=30$, respectively. (b) Response of the $\mathrm{CO}_{2}$ production rate to the applied pressure given in (a) for $k=0.01$ and $d=0.9$. The dotted line marked $\langle r\rangle$ indicates the long-time average of the period-averaged $\mathrm{CO}_{2}$ production rate $r$. In contrast to the case for $d=0$, this value cannot be distinguished from the maximum average $\mathrm{CO}_{2}$ production rate for constant $y=y_{2}(k, d)$, shown in Fig. 1 Time is measured in units of Monte Carlo steps per site.

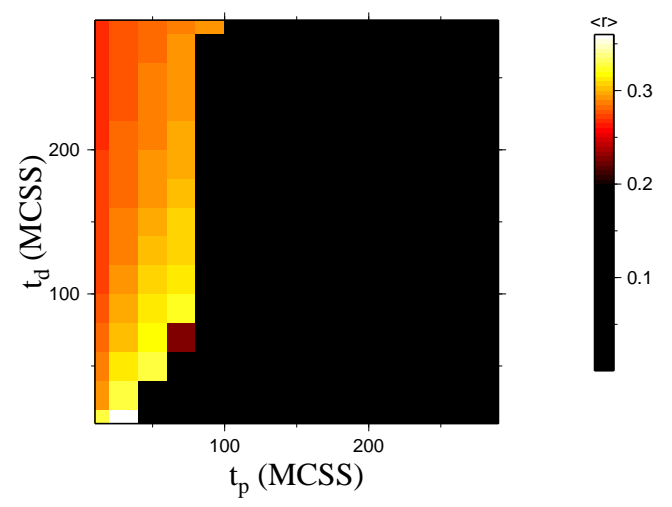

FIG. 4: Long-time average $\langle r\rangle$ of the period-averaged $\mathrm{CO}_{2}$ production rate, shown as a density plot vs $t_{d}$ and $t_{p}$ for $y_{l}=0.54$, $y_{h}=0.59, k=0.01, d=0.9$ and $L=100$. 